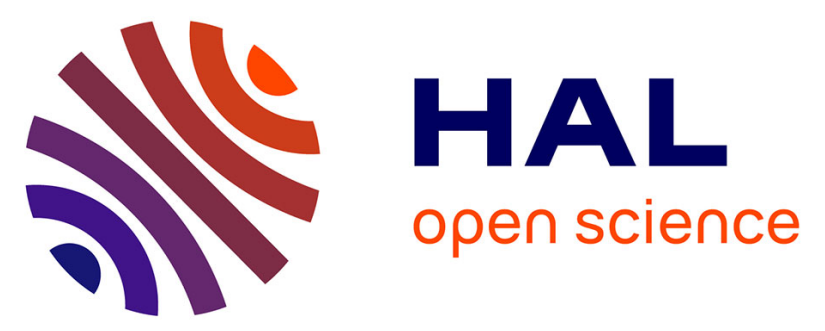

\title{
Experimental characterization of three-dimensional Graphene's thermoacoustic response and its theoretical modelling
}

Zhi Lin Ngoh, Pierre Guiraud, Dunlin Tan, Stefano Giordano, Olivier Bou Matar, Edwin Hang Tong Teo, Philippe Pernod, Philippe Coquet, Raphael Lardat

\section{To cite this version:}

Zhi Lin Ngoh, Pierre Guiraud, Dunlin Tan, Stefano Giordano, Olivier Bou Matar, et al.. Experimental characterization of three-dimensional Graphene's thermoacoustic response and its theoretical modelling. Carbon, 2020, 169, pp.382-394. 10.1016/j.carbon.2020.06.045 . hal-02947956

\section{HAL Id: hal-02947956 https://hal.science/hal-02947956}

Submitted on 28 Sep 2020

HAL is a multi-disciplinary open access archive for the deposit and dissemination of scientific research documents, whether they are published or not. The documents may come from teaching and research institutions in France or abroad, or from public or private research centers.
L'archive ouverte pluridisciplinaire HAL, est destinée au dépôt et à la diffusion de documents scientifiques de niveau recherche, publiés ou non, émanant des établissements d'enseignement et de recherche français ou étrangers, des laboratoires publics ou privés. 


\title{
Experimental Characterization of Three-Dimensional Graphene's Thermoacoustic Response and its Theoretical Modelling
}

\author{
Zhi Lin Ngoh ${ }^{1,2,3, \cdot}$, Pierre Guiraud ${ }^{2,4,5, \cdot}$, Dunlin Tan ${ }^{2,3}$, Stefano Giordano ${ }^{4, *}$, \\ Olivier Bou-Matar ${ }^{4}$, Edwin Hang Tong Teo ${ }^{1,2,6, *}$, Philippe Pernod ${ }^{4}$, Philippe Coquet \\ 2,4 , Raphael Lardat 5 \\ ${ }^{I}$ School of Electrical and Electronic Engineering, Nanyang Technological University, 50 Nanyang Avenue, 639798, Singapore \\ ${ }^{2}$ UMI 3288 CINTRA CNRS-NTU-THALES, Nanyang Technological University, Research Techno Plaza, 50 Nanyang Drive, 637553, \\ Singapore \\ ${ }^{3}$ Research \& Technology, Thales Solutions Asia Pte Ltd, 21 Changi North Rise, 498788, Singapore \\ ${ }^{4}$ Univ. Lille, CNRS, Centrale Lille, ISEN, Univ. Valenciennes, UMR 8520_IEMN, LIA LICS/LEMAC, F-59000 Lille, France \\ ${ }^{5}$ Thales Underwater System France, Sophia Antipolis, 525 Route des Dolines, F-06560 Valbonne, France \\ ${ }^{6}$ Advanced Materials, Temasek Laboratories@NTU, 50 Nanyang Drive, Singapore, 637553, Singapore \\ - Authors contributed equally to this work \\ *stefano.giordano@univ-lille.fr \\ *htteo@ntu.edu.sg
}

Elsevier use only: Received date here; revised date here; accepted date here

\begin{abstract}
In the past decade, a lot of research has been conducted on the potential of carbon nanostructured materials to emit sound via thermoacoustics through both simulations and experiments. However, experimental validation of simulations for three-dimensional graphene (3D-C), which has a complicated 3D structure, has yet to be achieved. In this paper, 3D-C is synthesized via thermal chemical vapor deposition and its microstructure and quality tested using Scanning Electron Microscopy and Raman spectroscopy respectively. Then, a two temperature model is used to predict the effects of numerous parameters: frequency, input power, sample size, connection area, connection path, pores per inch, thickness, compression as well as the addition of a backing on the acoustic performance and temperature of the
\end{abstract}


sample. The experimental results presented in this paper validate the predictions of the adopted two temperature model. The efficiency of 3D-C is then compared with results presented in other studies to understand how the presented 3D-C fared against ones from the literature as well as other carbon nanostructured materials.

Keywords: three-dimensional graphene (3D-C), thermoacoustics, chemical vapor deposition, theoretical modelling, loudspeaker

\section{Introduction}

Traditional mechanical methods of sound generation (using loudspeakers or piezoelectric devices), are inherently resonant devices whose resonance frequency is dependent on the size and shape of the driver. Furthermore, they utilize rare materials (magnets $[1,2]$ ), resulting in heavy and expensive devices. Thermophones are an alternative capable of generating wideband sound generation independent of material size. In addition, they do not use any moving parts, are lightweight and do not require the use of any rare or expensive material. The mechanism of thermoacoustics involves an electric alternating current (AC) being injected into a material and, due to the effects of Joule heating, a thermal gradient is generated within the material. Based on the laws of thermodynamics, the thermal gradient induces a pressure gradient in the surrounding medium, hence emitting sound [3-5]. This nonmechanical method of sound generation via thermoacoustics allows the material to emit sound in a wide frequency range independent of the material size. In order for the material to be able 
to emit sound using thermoacoustics, it should have a high thermal conductivity to allow for thermal energy to dissipate quickly from the material into the surrounding medium; and a low heat capacity per unit area (HCPUA) such that minimal heat is stored within the material during operation [6].

Since carbon nanostructured materials possess the thermal properties required [7], extensive research has been conducted on their potential to emit sound via the thermoacoustic effect, such as carbon nanotubes (CNTs) [6-19], 2D graphene [20-25], and 3D graphene (3DC) $[26,27]$. These studies demonstrate the influence of frequency, input power, microstructure and backing on the acoustics performance of the carbon nanostructured materials, obtained from the conducted experiments. An extensive theoretical analysis of the thermoacoustic sound generation in many different configurations is available [4, 16, 28-34]. However, due to the unique and complex microstructure of 3D-C, very few models have been adapted to fit its geometry precisely. Nonetheless, standard theoretical models have been used and display good correlation with experiments $[24,35,36]$. In addition, the freeze-dried 3D-C thermoacoustic investigations conducted by Fei et al. [26] and Lee et al. [27] only studied the effect of distance, frequency and input power on sound pressure level (SPL). The effect of an acoustical backing and variation of material properties and connection methods were left unexplored. Moreover, the presence of defects were detected in 3D-C utilized in previous studies and had electrical resistances more than $30 \Omega[26,27]$. Lastly, the fabrication process of $3 \mathrm{D}-\mathrm{C}$ is an inexpensive and easily scalable method for thermophone fabrication [37] compared to other proposed techniques. For this reason, it is important to further investigate the thermoacoustic 
properties of 3D-C which have been considered in only a limited number of research activities.

In this study, we investigate the suitability of Guiraud et al.'s simulation model [38] for describing the acoustic performance of 3D-C. First 3D-C was synthesized in-house and the microstructure and quality of the material tested with the use of a Scanning Electron Microscope (SEM) and Raman spectroscope respectively. Then, acoustic measurements were performed to assess the acoustic performances of 3D-C. The influence of parameters, namely the electrical input frequency, input power, material size, area of electrical connection, path of electrical connections, material density, and material pore per inch (ppi) on SPL and temperature were investigated. Methods to improve the acoustics performance of $3 \mathrm{D}-\mathrm{C}$ were also explored. The obtained results were then compared to Guiraud et al.'s simulations, and other experimental results from literature [7, 26, 27].

\section{Thermoacoustic Theory}

In this section, we introduce the thermoacoustic model adopted to analyze the

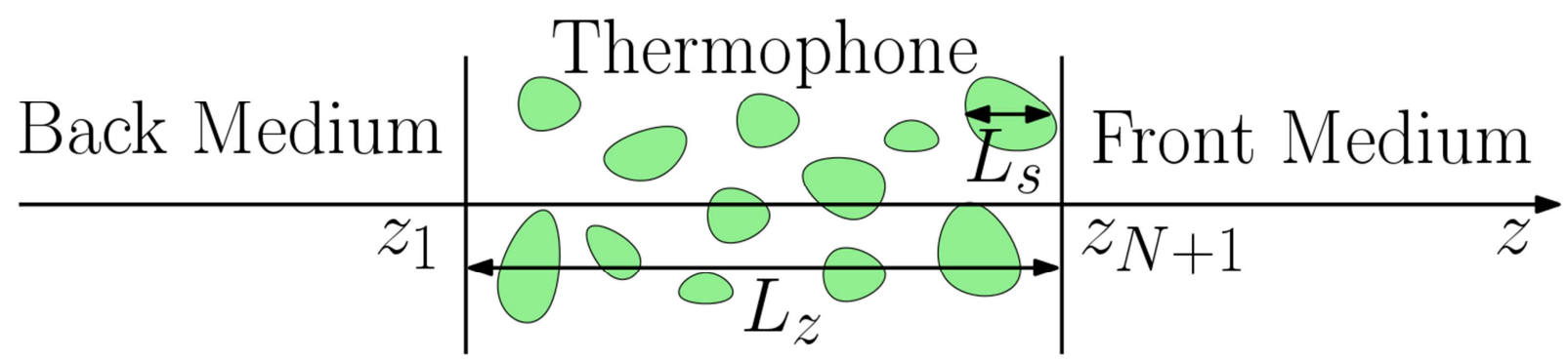
thermophone efficiency and the acoustic diffraction theory used for the far field estimations.

\subsection{Thermophone model}

Fig. 1. Schematic of the thermophone. $L_{z}$ and $L_{s}$ represent overall thickness of the material and mean diameter of the pore respectively. 
In order to have a better understanding of the experimental results, they will be compared to a theoretical model based on a "two temperature" assumption [39, 40]. Due to the thickness and the high porosity of 3D-C (more than 90\%), regular continuous thermoacoustic models cannot be used. The following model assumes that, for a thermophone radiating in free field, the solid (3D-C) and the fluid (air) coexists in the sample as seen in Fig. 1. Due to the high frequency of the electrical energy supplied to the thermophone, the local thermal equilibrium is not attained between the two phases. Therefore, the general conservation equations for mass, linear momentum and energy in a fluid [41, 42]

$\frac{1}{B_{0}} \frac{\partial p}{\partial t}-\alpha_{T} \frac{\partial T}{\partial t}+\vec{\nabla} \cdot \vec{v}=0$

$\rho \frac{\partial \vec{v}}{\partial t}=-\vec{\nabla} p+\mu \nabla^{2} \vec{v}+(\lambda+\mu) \vec{\nabla}(\vec{\nabla} \cdot \vec{v})$

$\rho C_{p} \frac{\partial T}{\partial t}-\alpha_{T} T_{0} \frac{\partial p}{\partial t}=\kappa \nabla^{2} T$

can be coupled with the energy conservation for the solid phase using a different temperature

$\rho_{s} C_{V, s} \frac{\partial T_{s}}{\partial t}=\kappa_{s} \nabla^{2} T_{s}+S_{0}$

whereby the pressure $p$ (Pascal), the temperatures of the fluid and solid $T, T_{S}$ (Kelvin) respectively and the particle velocity $\vec{v}(\mathrm{~m} / \mathrm{s})$ are the main variables depending on time $t$ (seconds) and the position vector $\vec{r}$ (meters). Otherwise, $\rho$ is the density $\left(\mathrm{kg} / \mathrm{m}^{3}\right), B_{0}$ is the bulk modulus (Pa), $\alpha_{T}$ is the coefficient of volumetric expansion $(1 / \mathrm{K}), \lambda$ and $\mu$ are the first and second viscosity coefficients (Pa.s), $C_{p}$ and $C_{V, s}$ are the specific heat at constant pressure of the fluid and at constant volume of the solid respectively $[\mathrm{J} /(\mathrm{kg} . \mathrm{K})], T_{0}$ is the ambient temperature (Kelvin), $\kappa$ is the thermal conductivity $[\mathrm{W} /(\mathrm{m} . \mathrm{K})]$ and, lastly, $S_{0}$ is the input 
power density $\left(\mathrm{W} / \mathrm{m}^{3}\right)$. Considering, for simplification purposes, a $1 \mathrm{D}$ model (plane wave radiation) with harmonic time dependence, Eqs. (1) to (4) can be rewritten as

$\frac{i \omega}{B_{0}} p-i \omega \alpha_{T} T+\frac{d v}{d z}=0$,

$i \omega \rho v=-\frac{d p}{d z}+(\lambda+2 \mu) \frac{d^{2} v}{d z^{2}}$

$i \omega \rho C_{p} T=\kappa \frac{d^{2} T}{d z^{2}}+i \omega \alpha_{T} T_{0} p$

$i \omega \rho_{s} C_{v, s} T_{S}=\kappa_{s} \frac{d^{2} T_{s}}{d z^{2}}+S_{0}$,

respectively, whereby $\omega$ is the radial frequency, $i$ is the imaginary unit, and $z$ is the space variable.

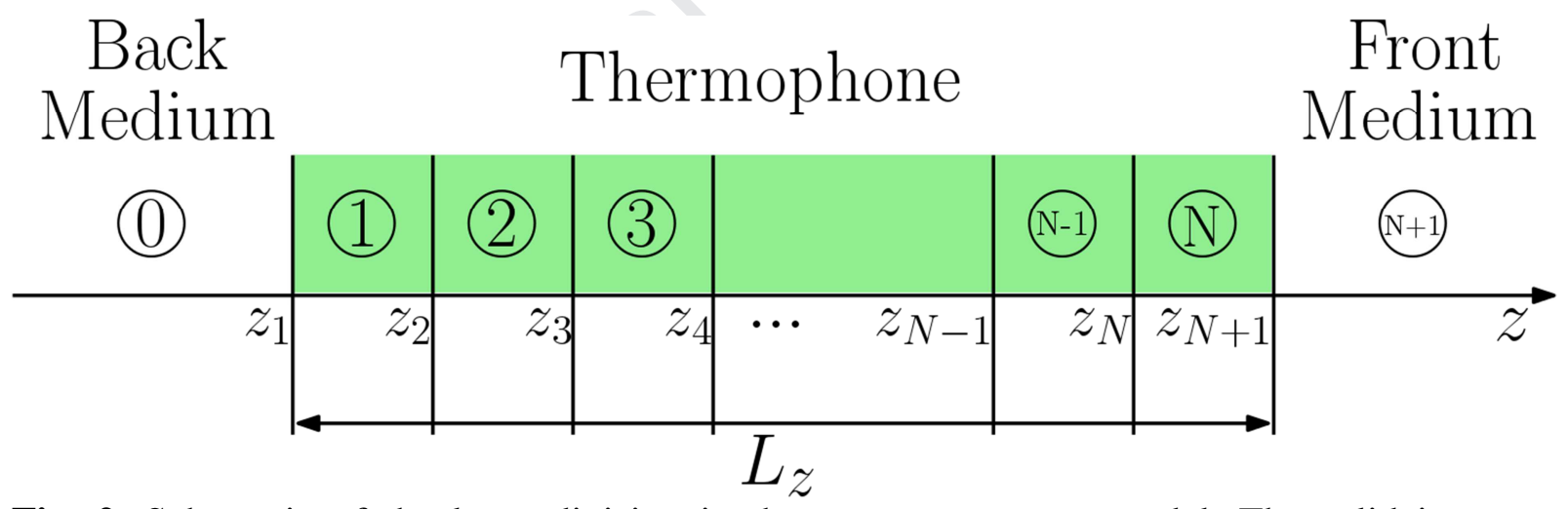

Fig. 2. Schematic of the layer division in the two temperature model. The solid in green coexist with its surrounding fluid in layers 1 to $N$. Surrounding fluid is present in layers 0 to $N+1$.

In order to simulate the transfer of energy from the solid to the fluid, necessary for the thermoacoustic generation, the $3 \mathrm{D}-\mathrm{C}$ is subdivided into $N$ discretized layers as seen in Fig. 2 
in which both the solid and the fluid coexists. At the layers' interfaces, normal surface tension, velocity and temperatures (both for solid and fluid) are considered continuous. However, for the thermoacoustic generation to occur, a fluid/solid coupling parameter $g\left[\mathrm{~W} /\left(\mathrm{m}^{2} . \mathrm{K}\right)\right]$ proportional to the difference of temperature of the two phases, is introduced in the continuity condition of the heat flux. The boundary conditions at such an interface are written as

$\tilde{p}_{j-1}\left(z_{j}\right)=\tilde{p}_{j}\left(z_{j}\right)$

$v_{j-1}\left(z_{j}\right)=v_{j}\left(z_{j}\right)$,

$T_{j-1}\left(z_{j}\right)=T_{j}\left(z_{j}\right)$,

$T_{s, j-1}\left(z_{i}\right)=T_{s, j}\left(z_{j}\right)$,

$q_{j}\left(z_{j}\right)=q_{j-1}\left(z_{j}\right)+g\left(T_{s, j}\left(z_{j}\right)-T_{j}\left(z_{j}\right)\right)$,

$q_{s, j}\left(z_{j}\right)=q_{s, j-1}\left(z_{j}\right)-g\left(T_{s, j}\left(z_{j}\right)-T_{j}\left(z_{j}\right)\right)$,

for all $j \in[0, N+1]$ discretized layers with $q_{s, 1}\left(z_{1}\right)=q_{s, N+1}\left(z_{N+1}\right)=0$. Here, we define the normal surface tension in the fluid as $\tilde{p}=p-(\lambda+2 \mu) \frac{d v}{d z}$, the fluid heat flux as $q=$ $-\kappa \frac{d T}{d z}$ and the solid heat flux as $q_{s}=-\kappa_{s} \frac{d T_{s}}{d z}$. Eqs. (13) and (14) represent the transfer of thermal energy from the solid to the fluid, controlled by the parameter $g$.

Explicit equations for $\tilde{p}, v, T$ in any fluid medium are derived in Eqs. (A.2, A.7) and (A.9) in the supporting information. We introduce the functions $G^{(a)}$ and $G^{(b)}$ as well as the constants $\mathrm{L}_{1}$ and $\mathrm{L}_{2}$ as

$G^{(a)}(\eta)=\alpha_{T} B_{0}-\left(\frac{B_{0}}{i \omega}+\lambda+2 \mu\right)\left(L_{1} \eta^{2}+L_{2} \eta^{4}\right)$, 
$G^{(b)}(\eta)=L_{1}+L_{2} \eta^{2}$

$L_{1}=-\frac{1}{i \omega \rho}\left(\alpha_{T} B_{0}+\frac{i \omega \rho C_{V}}{\alpha_{T} T_{0} B_{0}}\left(\lambda+2 \mu+\frac{B_{0}}{i \omega}\right)\right)$,

$L_{2}=\left(\lambda+2 \mu+\frac{B_{0}}{i \omega}\right) \frac{\kappa}{i \omega \rho \alpha_{T} T_{0} B_{0}}$,

as well as the matrices

$H^{(a)}=\left[\begin{array}{cccc}G^{(a)}\left(i k_{a c}\right) & G^{(a)}\left(i k_{a c}\right) & G^{(a)}\left(\theta_{t h}\right) & G^{(a)}\left(\theta_{t h}\right) \\ -i k_{a c} G^{(b)}\left(i k_{a c}\right) & i k_{a c} G^{(b)}\left(i k_{a c}\right) & -\theta_{t h} G^{(b)}\left(\theta_{t h}\right) & \theta_{t h} G^{(b)}\left(\theta_{t h}\right) \\ \kappa i k_{a c} & -\kappa i k_{a c} & \kappa \theta_{t h} & -\kappa \theta_{t h} \\ 1 & 1 & 1 & 1\end{array}\right]$,

$H^{(b)}(z)=\left[\begin{array}{cccc}e^{-i k_{a c} z} & 0 & 0 & 0 \\ 0 & e^{i k_{a c} z} & 0 & 0 \\ 0 & 0 & e^{-\theta_{t h z}} & 0 \\ 0 & 0 & 0 & e^{\theta_{t h} z}\end{array}\right]$.

where $k_{a c}$ and $\theta_{t h}$ represent positive fluid acoustical wavenumber and thermal wavenumber respectively.

The defining variables $\tilde{p}, v, q, T$ in fluid medium are then written in any layer $j \in[0, N+1]$ in a matrix form as

$\left[\begin{array}{l}\tilde{p}_{j}(z) \\ v_{j}(z) \\ q_{j}(z) \\ T_{j}(z)\end{array}\right]=H^{(a b)}(z)\left[\begin{array}{l}A_{j} \\ B_{j} \\ C_{j} \\ D_{j}\end{array}\right]$

with $H^{(a b)}(z)=H^{(a)} H^{(b)}(z)$. Similarly, an equation for $T_{S}$ is found in Eq. (A.12) (Supporting Information) and can be rewritten as

$H^{(c)}(z)=\left[\begin{array}{cc}\kappa_{s} \theta_{\text {solid }} e^{-\theta_{\text {solid } z}} & -\kappa_{s} \theta_{\text {solid }} e^{\theta_{\text {solid }}} \\ e^{-\theta_{\text {solid }}} & e^{\theta_{\text {solid }} z}\end{array}\right]$, 
$\left[\begin{array}{l}q_{s, j}(z) \\ T_{s, j}(z)\end{array}\right]=H^{(c)}(z)\left[\begin{array}{l}E_{j} \\ F_{j}\end{array}\right]+\left[\begin{array}{c}0 \\ T_{S, 0}\end{array}\right]$.

$T_{S, 0}$ is the particular solution of the differential equation associated to $T_{S}$ and represent the input of energy in the solid. $\theta_{\text {solid }}$ represent positive thermal wavenumber in the solid. The coefficient $A, B, C, D, E, F$ are unknown constants different in each layer that, if determined, allowed for full determination of the parameters $\tilde{p}, v, q, T, q_{s}, T_{s}$ at any position and frequency. Therefore, using the boundary conditions described in Eqs. (9) to (14), this is a system of $6(N+2)$ unknowns and $6(N+2)$ boundary conditions, where $N$ is still the number of discretized layers. This well posed problem allowed to fully determine all unknowns (see Supporting Information A). Thermoacoustic generation of a thick porous media is then estimated in the near field of the sample and all the parameters $\tilde{p}, v, q, q_{s}, T, T_{s}$ can be evaluated at any position and frequency. More explanations regarding the equations and the development of the model can be found in Guiraud et al. $2019[34,38]$ and the derivation section in Supporting Information.

\subsection{Acoustic diffraction in the far field}

In order to investigate experimental results in the far field, the acoustical diffraction due to the finite size of the sample was taken into consideration. Since 3D-C has a complex geometry, several approximations were made. Using the 1D two temperature model described in sub-section 2.1, the pressure and the particle velocity of the foam at the limit of the thermal layer, is evaluated. For a sample generating thermoacoustic waves, the thermal layer is the length of fluid in the vicinity of the surface in which the variation of temperature will create 
the acoustical wave. This thermal layer can be estimated from Eq. (A.11) (Supporting Information) assuming weak viscosity as $[28,34,38]$

$L_{t h}=\sqrt{\frac{2 \kappa}{\rho C_{p} \omega}}$.

At this distance, the acoustical wave is fully generated and this can be considered as the

top of the equivalent fluid piston [14]. We then assimilate this position as an equivalent radiating

plate to use the Rayleigh's integral for far field diffractions as

$p_{F F}(x, y, z)=\frac{i \omega \rho}{4 \pi} \int_{-L_{x} / 2}^{L_{x} / 2} \int_{-L_{y} / 2}^{L_{y} / 2} v\left(x^{\prime}, y^{\prime}\right) \frac{e^{-i k r}}{r} d x^{\prime} d y^{\prime}$

$r=\sqrt{\left(z-L_{t h}\right)^{2}+\left(y-y^{\prime}\right)^{2}+\left(x-x^{\prime}\right)^{2}}$

with $v\left(x^{\prime}, y^{\prime}\right)$ the velocity at $L_{t h},(x, y, z)$ the recording position, $\left(x^{\prime}, y^{\prime}, L_{t h}\right)$ the generating point position and $L_{x}, L_{y}$ the size of the sample, whereby $L_{x}$ is the length between the electrical contacts. The sound pressure level (SPL) in $\mathrm{dB}$ is then estimated as 
$S P L=20 \log _{10}\left(\frac{p_{r m s}}{p_{r e f}}\right)$

with $\boldsymbol{p}_{\text {ref }}$ being $20 \boldsymbol{\mu} \mathrm{Pa}$ in air.

\section{Results and discussion}

In this section the experimental characterizations of the samples are discussed, and their characteristics explained. The samples are then acoustically and thermally tested and the results are presented and analyzed.

\subsection{Material characterization}

3D-C was synthesized as described in the experimental section in supporting information and the visual image (Fig. 3a) and SEM micrograph (Fig. 3b) were obtained. The SEM micrograph of the obtained free-standing 3D-C indicates that the microstructure of the synthesized 3D-C did not collapse and instead has similar microstructures as that of freezedried 3D-C synthesized by Fei et al. and Lee et al. [26, 27]. Raman spectroscopy was conducted on 3D-C to determine its crystallinity and the obtained spectrum can be seen in Fig. 3c. Only 2 peaks appear at $1580 \mathrm{~cm}^{-1}$ and $2705 \mathrm{~cm}^{-1}$, which represent the $\mathrm{G}$ and $2 \mathrm{D}$ peaks respectively, indicating the presence of graphene. The absence of the defect peak at $1350 \mathrm{~cm}^{-1}$ indicates that the graphene present in 3D-C were of pristine quality [26, 43-47]. 
(a)

(b)
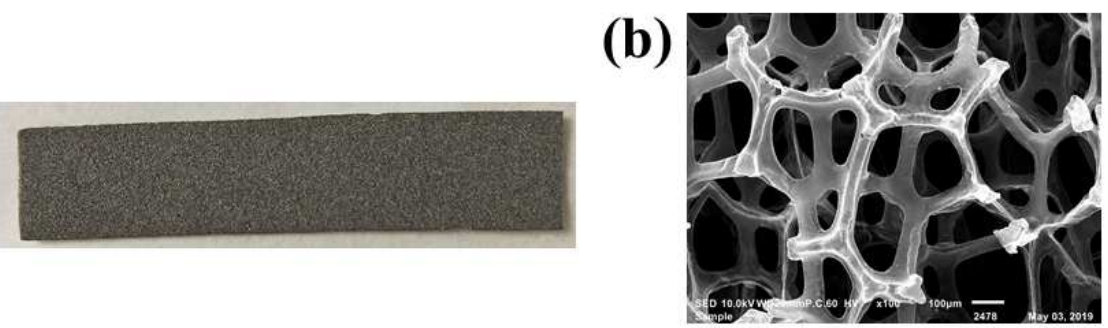

(c)

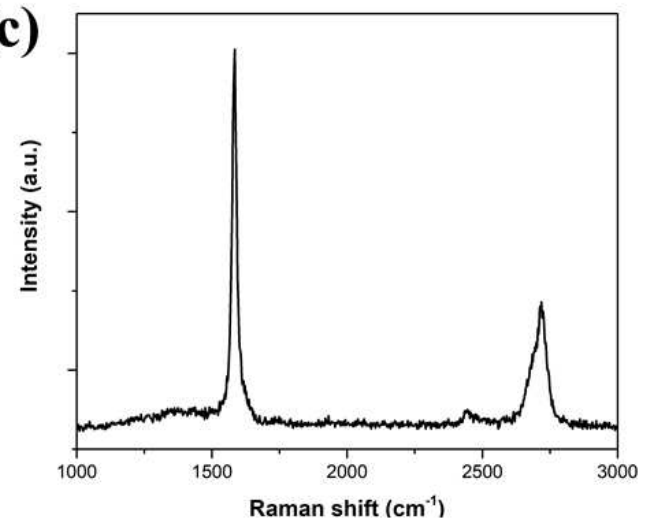

Fig. 3. Chemical characterization of 3D-C. (a) Visual image of 3D-C; (b) SEM micrograph of 3D-C; (c) Raman spectrum of 3D-C. 


\subsection{Acoustical measurements}

The geometries of the samples used and the physical parameters of the medium (air and 3D-C) can be found in Table S1 and Table S2 respectively. All measurements were done with recording distances of $3 \mathrm{~cm}$ to improve the signal to noise ratio but the reported SPL are normalized to a recording distance of $1 \mathrm{~m}$. All reported frequency spectrum measurements are also normalized with an input power of $1 \mathrm{~W}$. As indicated in the acoustic measurement set-up in supporting information, all measurements were made using only an alternating current (AC) signal generator. Without a DC supply, a thermophone will radiate at twice the generating its generating frequency. It is physically understood as, when an AC signal runs through the sample, the temperature is unable to have negative values, resulting in its variation being proportional to twice the frequency of the AC signal. Mathematically speaking, the sound pressure is proportional to the input power given by [48]

$P_{\text {input }}=\frac{V_{\text {input }}^{2}}{R}=\frac{\left(V_{A C} \cos \omega t\right)^{2}}{R}=\frac{V_{A C}{ }^{2}}{2 \mathrm{R}}(1+\cos 2 \omega t)$

Hence, the presented acoustic spectra correspond to the acoustical frequency, the first harmonics of the AC input. Power measurements and the associated recorded temperatures were obtained at an AC input frequency of $5 \mathrm{kHz}$, which induces an acoustical wave at $10 \mathrm{kHz}$. 
(a)

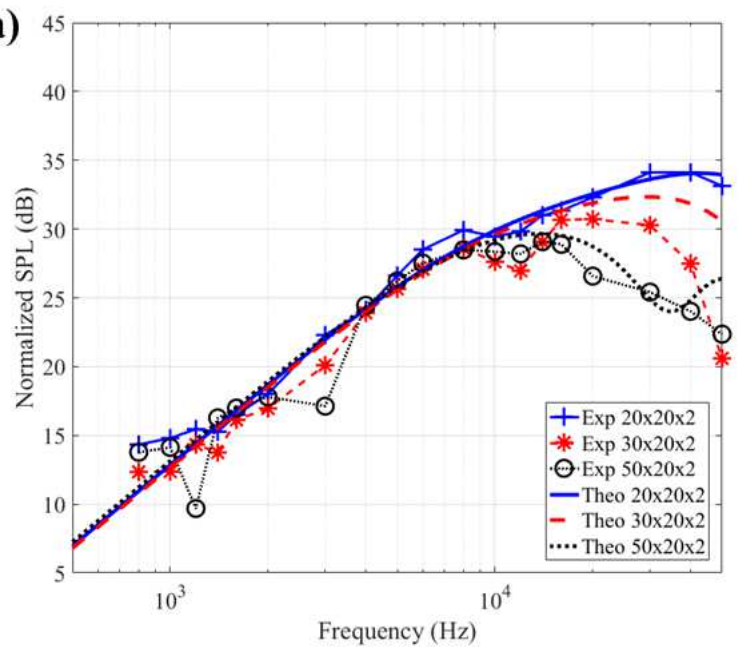

(b)

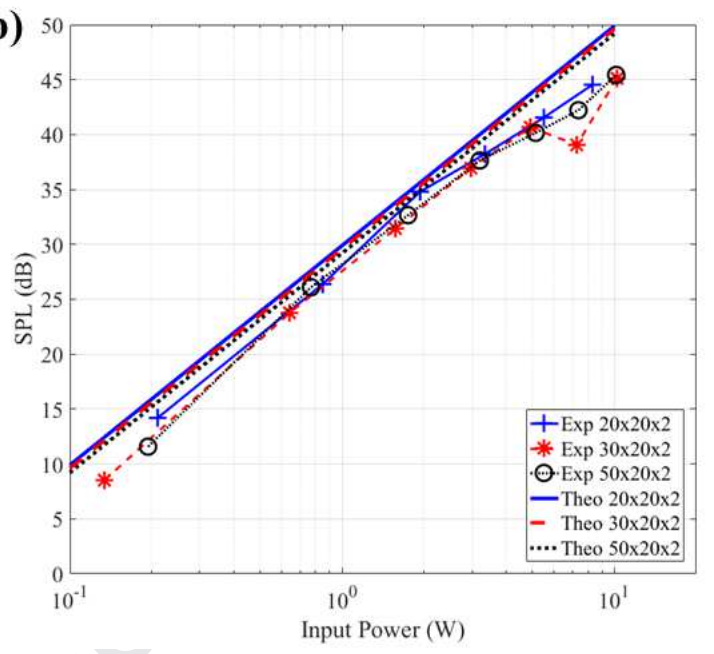

Fig. 4. (a) Acoustical frequency and, (b) power spectrum of 3D-C of different sizes in millimeter.

\section{2. 1 Effect of sample size}

Fig. 4 shows the frequency and power spectrum of three of the samples with the same intrinsic parameters but of different sizes (see details in legend of Fig. 4). From Fig. 4b, we deduce that the SPL and the input power exhibit a linear relationship, both experimentally and theoretically. Similarly, in Fig. 4a there is a linear relationship (20dB/decade) between SPL and frequency from $1 \mathrm{kHz}$ to $\sim 10 \mathrm{kHz}$. This linear behaviour agrees with the thermoacoustic theory and the recent literature $[16,23,28,33]$. In the higher frequency range, from $\sim 10 \mathrm{kHz}$ to $50 \mathrm{kHz}$, acoustic diffraction is observed. The longest sample has a lower anti resonance frequency, resulting in a decrease in SPL before the other samples. Since this classical acoustical result fits our theoretical curve, the assumption made in sub-section 2.2 of 
considering a planar diffraction with the velocity taken at the limit of the thermal layer is then validated. Sample length affects the electrical DC resistance of the sample (Table B.2). Indeed, due to the longer path which the electrical current has to go through ( $L_{x}$ in Table B.2), longer samples display higher DC resistance. This however does not influence the overall SPL of the sample as both frequency and power spectra are in the same range for the three sample with different size. Since thermophones are driven by thermal power and not electrical potential, different DC resistances can create impedance matching issues with the interfacing hardware but will not change the acoustic radiation once normalized. However, it was also observed that for the same input power, the temperature was the lowest for the longest sample. This is due to the increased surface area which the sample has, allowing thermal energy to dissipate into the surrounding medium at a faster rate. With increased heat dissipation, the observed temperatures are lower.

\subsubsection{Effect of connections}

\subsubsection{Connection area}

(a)

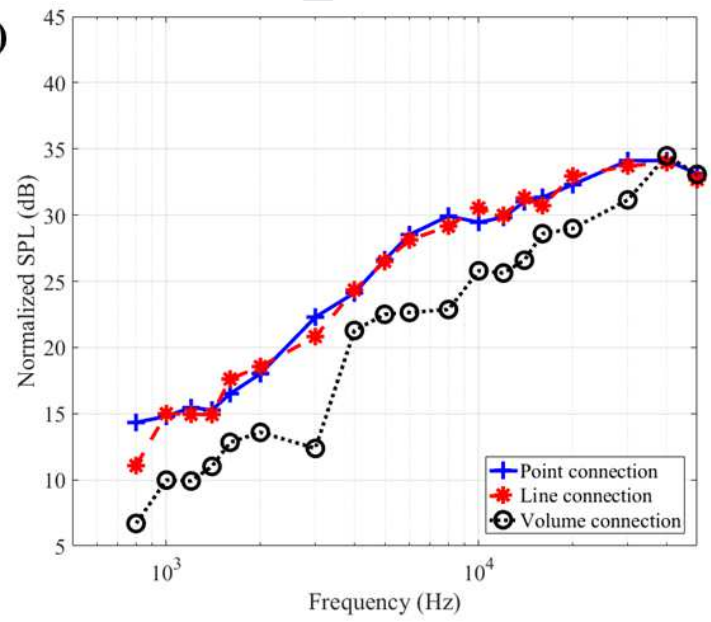

(b)

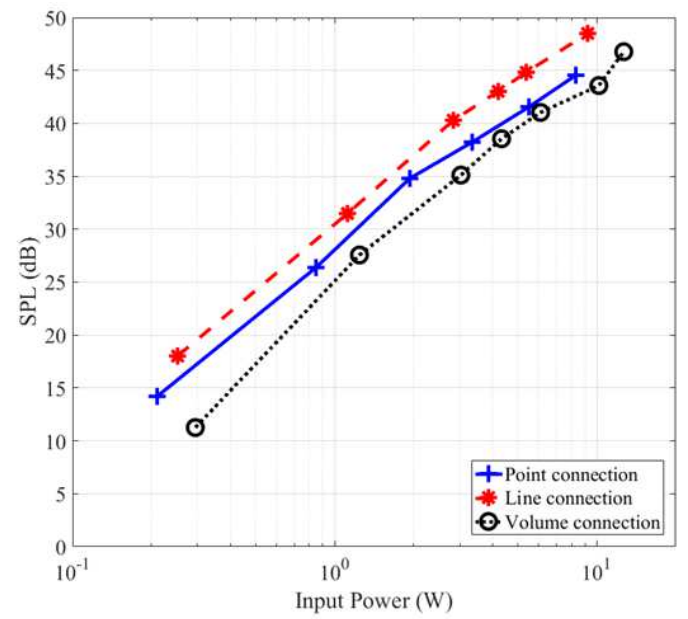

Fig. 5. (a) Acoustical frequency and, (b) power spectrum of samples of the same size but with different silver paste connections, see Table B.1. 
(a)

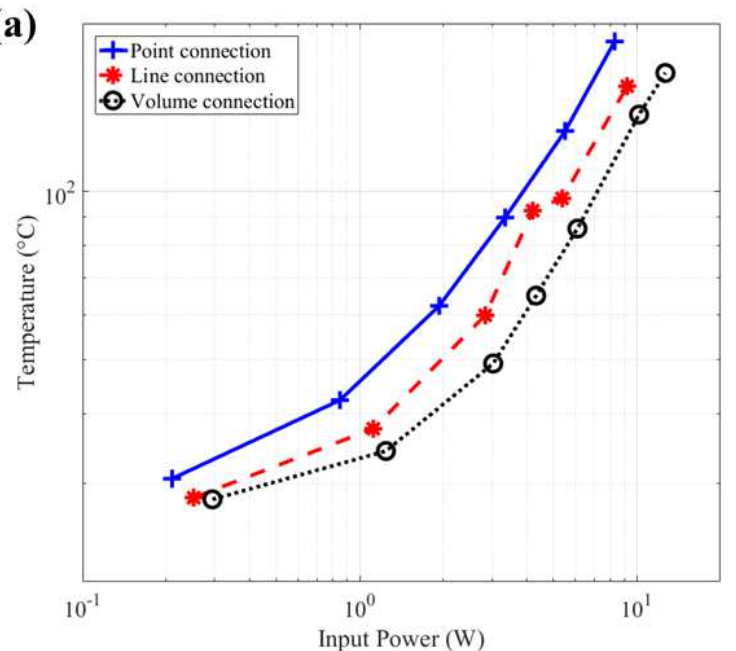

(b)

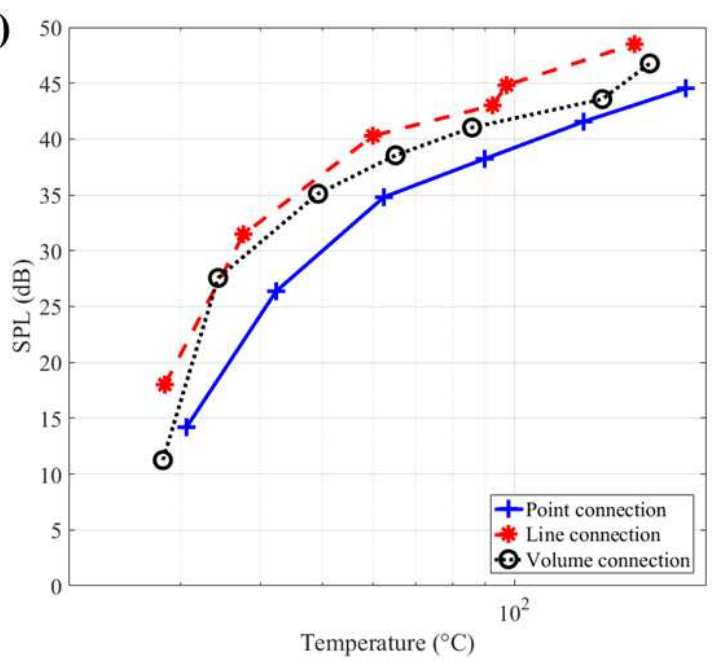

Fig. 6. Temperature dependence of the samples with different silver paste connections. (a)

Relationship between temperature and input power and, (b) relationship between SPL and temperature.

Fig. 5 and Fig. 6 show the results for samples of similar parameters and dimensions but with a difference in the way the connection was made. As seen in Table B.1, the point connection consists of just a dot of silver on the centre of the edges of 3D-C, the line connection has conductive silver paint following the edges of the $3 \mathrm{D}-\mathrm{C}$, and the volume connection has conductive silver paint dripped directly on $3 \mathrm{D}-\mathrm{C}$ to completely coat the thickness of 3D-C.

In the frequency spectrum of Fig. 5a, the point and line connection display similar results unlike the volume connection, which performs on average $5 \mathrm{~dB}$ lower across the whole frequency range. The thermal camera image in Fig. 7 shows that the silver paste is not heated, thus preventing the thermoacoustic process from happening on the edges of 3D-C. This resulted in a thermal energy loss, consequently decreasing its acoustical performance. The 
differences are not as pronounced in Fig. $5 \mathrm{~b}$ but it is seen that for a fixed input power the volume connection displays the lowest SPL. Unlike in Fig. 5a, it is seen that the line connection performs $2 \mathrm{~dB}$ higher than the point connection in Fig. $5 \mathrm{~b}$. This could be attributed

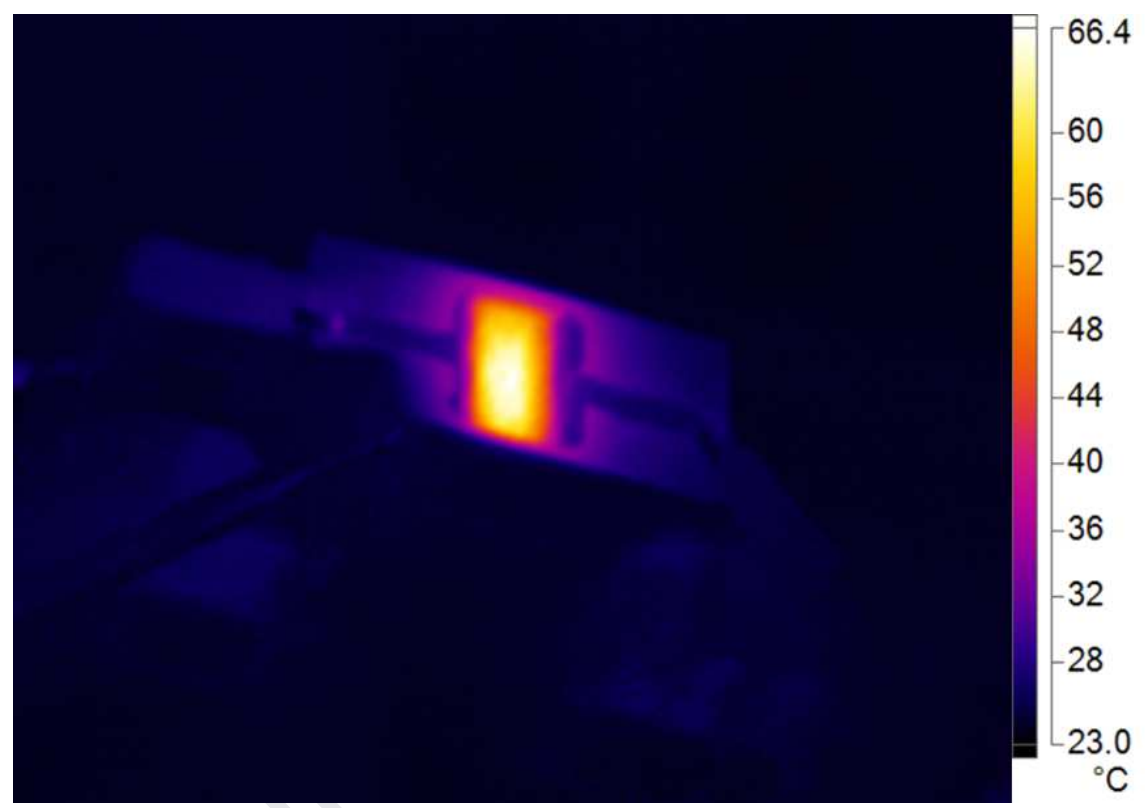

Fig. 7. Thermal image of 3D-C with volume connection with $3.3 \mathrm{~W}$ power input at an acoustical frequency of $10 \mathrm{kHz}$.

to measurements errors in either Fig. 5a or b but would lead to think that the line connection is more efficient than the point one.

In Fig. 6a, the thermal behaviour of 3D-C with increasing power is shown. Above $1 \mathrm{~W}$ of input power, the temperature increases linearly with input power, as also observed in the literature [7]. At low input power below $2 \mathrm{~W}$, the temperatures of the samples are similar to the temperature of the surrounding air medium, and no conclusion can be drawn on the linear relationship between the input power and temperature. More low power measurements have to 
be performed with different experimental conditions to be sure of the linearity between temperature and input power at all power levels. Fig. 7a shows that at the same input power, the point connection has a higher temperature than the line connection, which also has a higher temperature than the volume connection. Increased electrical contact surface with the sample decreases the power density in these regions, diminishing the hot point temperature. In Fig. 6b, the SPL is plotted against temperature. Although the normalized SPL of the point connection is $5 \mathrm{~dB}$ higher than the volume connection in Fig. 5a, the volume connection performed better in absolute SPL values relative to temperature in Fig. $6 b$.

In other words, Fig. 5a indicates that the line or point connection are equally as efficient, and both perform better than the volume connection, as indicated in Fig. $5 \mathrm{~b}$ as well. However, Fig. 6a and $\mathrm{b}$ show that the point connection is performing worse in terms of temperature than the others and is hence more likely to decompose prior to the other samples when a high input power is channelled into the sample. Hence, the better power repartition of the sample connected via line connection inflicts less heat damages when a high input power is injected into the sample, indicating its better resilience to high input power. 


\subsubsection{Connection path}

(a)

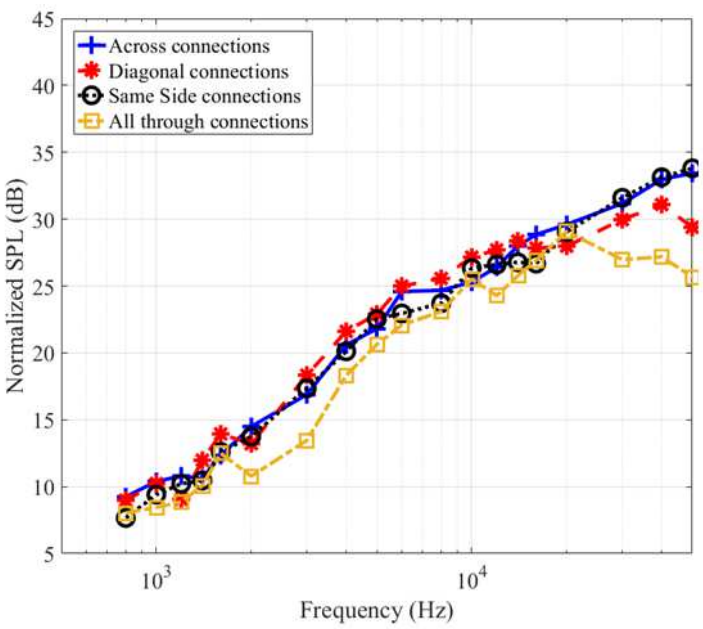

(b)

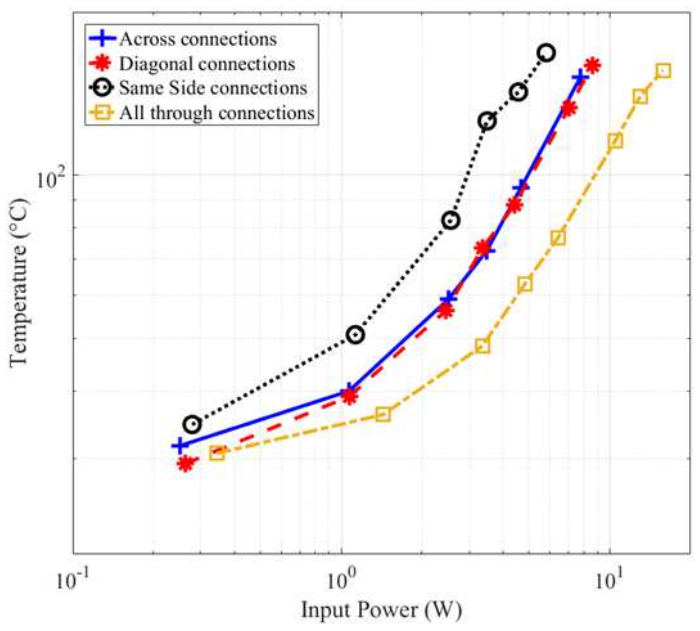

Fig. 8. (a) Acoustical frequency spectrum and (b) temperature variations of one sample with different electrical connection paths.

In Fig. 8, a single sample with four different connection points is investigated. The electrical path used changed for each measurement. Following the schematic of the sample seen in Table B.1, the paths investigated are 1-3 (across), 1-4 (diagonal), 1-2 (same side) and 12-34 (all through). As expected, changes in the path also lead to changes in the DC resistance as seen in Table B.2. The DC resistance of the path taken is directly proportional to the travel distance in 3D-C between the electrical contacts and their surface areas. Shorter paths have lower DC resistance, and increased contact surface results in lower DC resistance as well (Table B.2). Thermal images in Fig. 9 show that the temperature hot spots follow the least resistive path and that the temperature is most uniform only when the current is more uniformly distributed throughout the sample ("all through" connection). 

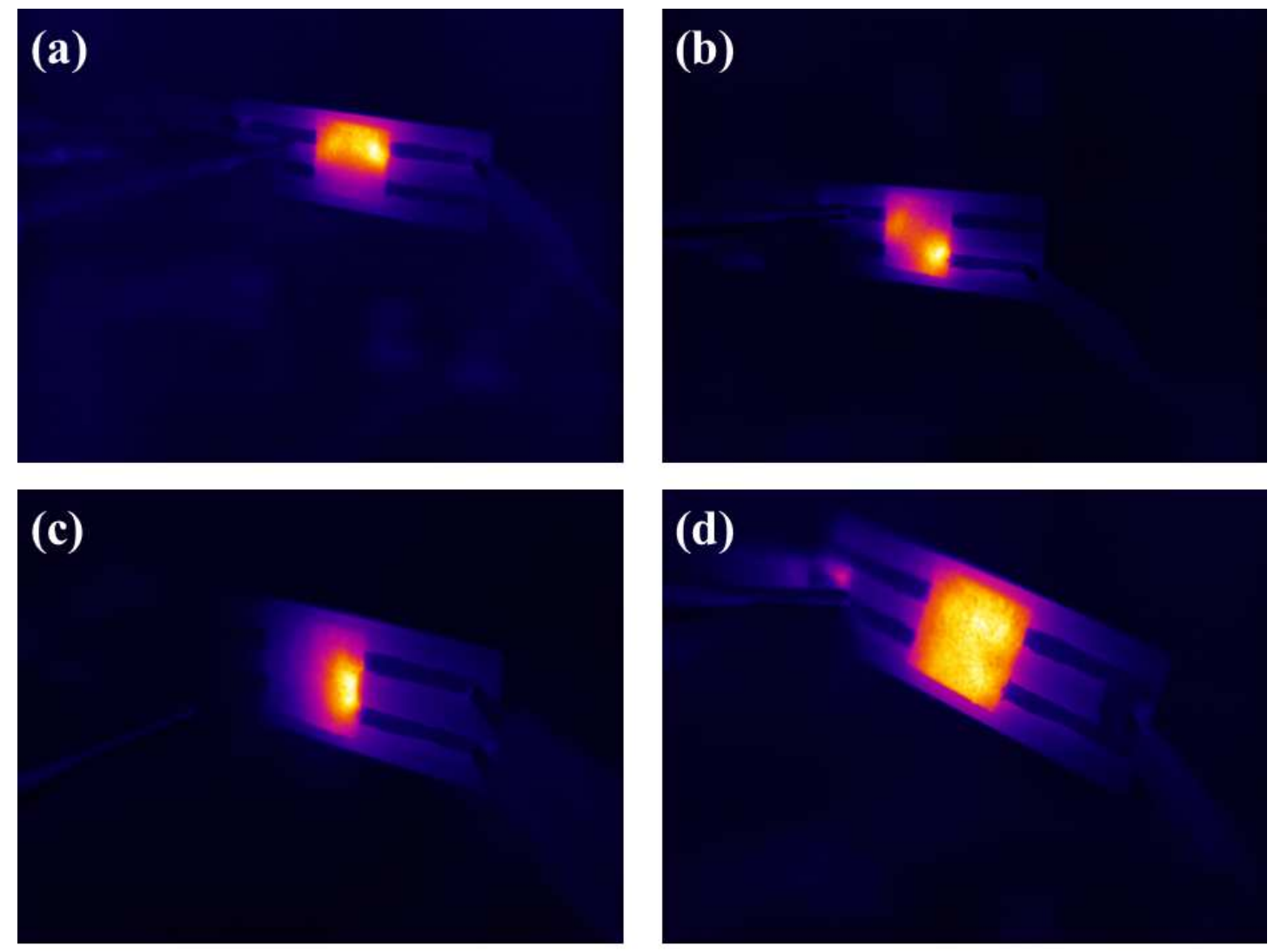

Fig. 9. Thermal images of the sample with multiple connections (a) across (b) diagonal (c) same side (d) all through.

Fig. 8a shows that at frequencies below $20 \mathrm{kHz}$, the samples' SPL have a linear relationship with the frequency. Despite the hot spots being located at the electrical connections, it is expected that high thermal conductivity of the samples would cause the entire 3D-C material to radiate. A single sample would then display similar normalized SPL regardless of the electrical path chosen, which is confirmed by the consistent spectra of Fig. 10a up to $20 \mathrm{kHz}$. At frequencies above $20 \mathrm{kHz}$, acoustic diffraction phenomena appear for all 
connection paths. If the entirety of 3D-C was radiating equally, the frequency spectra would be similar across the whole frequency range. However, these acoustic diffractions indicate that the acoustic radiation of the thermophone is dependent on the least resistive path. Due to the non-uniform distribution of energy, the entirety of 3D-C was not used to its maximum potential. Paradoxically, using the 3D-C in its "most efficient" configuration (uniform energy distribution) will create acoustic diffraction at lower frequencies due to the use of a longer radiating surface. This is seen in Fig. 9d with the "all through" path.

Comparing the results in Fig. $8 \mathrm{~b}$, the sample with the least resistive path (shortest travel distance, across connection) has a higher power density concentration. Hence, for a fixed input power, the temperature of the sample's hot spot will be higher than in a more distributed configuration (all through connection). The connection utilizing the most of the sample's surface and having a high connecting surface would hence have the highest power resilience. Connection "all through" is then recommended and concurs with the line connection analysis from sub-section 3.2.2.1 for samples with varying connection types (Fig. 6a).

\subsubsection{Effect of physical parameters of $3 D-C$}

Various physical parameters, namely the pore per inch (ppi), thickness and density of 3D-C, were varied and their influence on the acoustics performance investigated. 


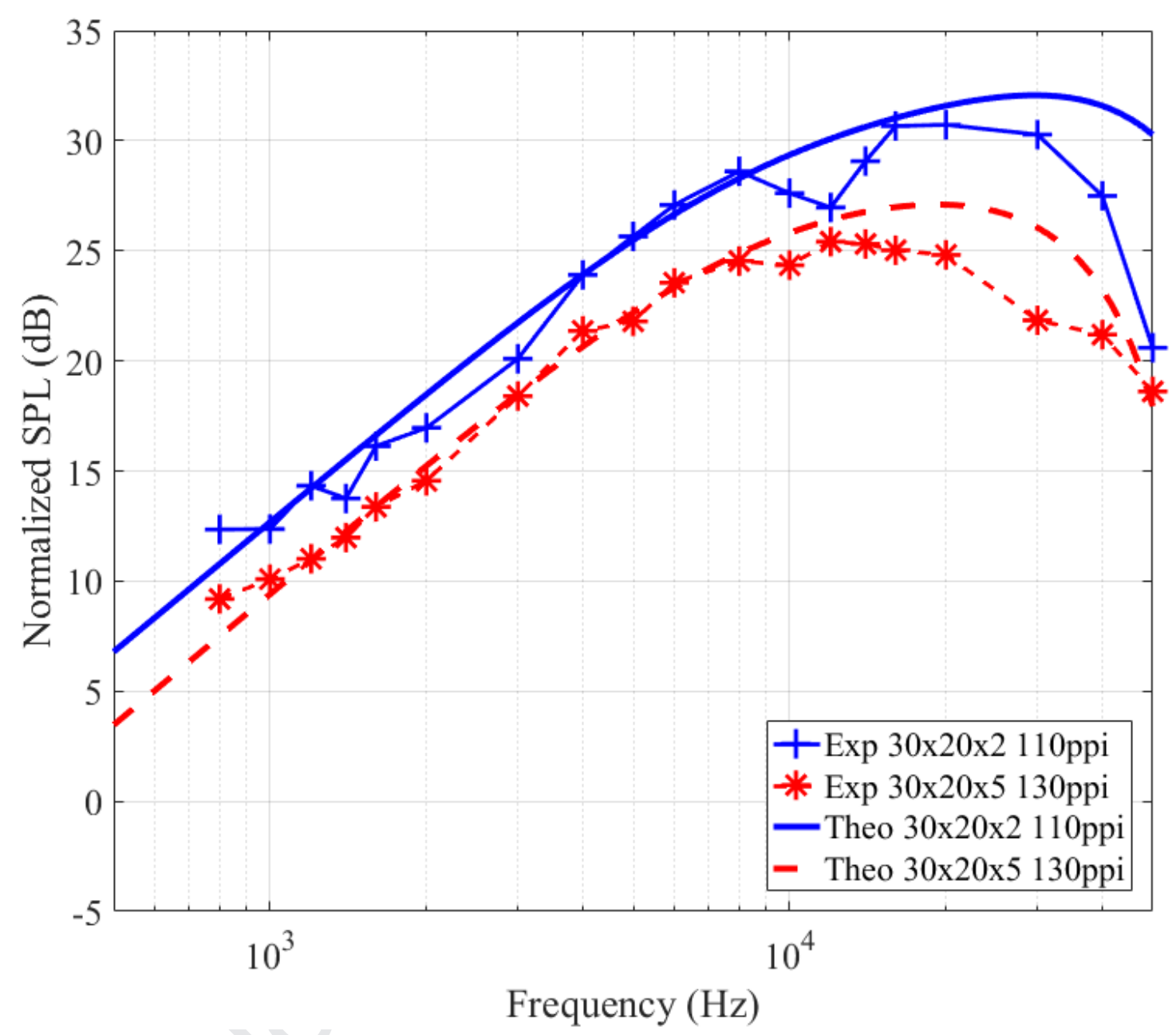

Fig. 10. Acoustical frequency spectrum of samples with different thickness and ppi.

Fig. 10 compares a regular sample $2 \mathrm{~mm}$ thick with $110 \mathrm{ppi}$ and a thicker $5 \mathrm{~mm}$ sample with 130 ppi. In their associated theoretical models, despite the difference in ppi, the change of thickness lead to the use of a similar number of layers per millimeter in both cases (Table B.2). Across the whole frequency range, there is a difference of $\sim 3 \mathrm{~dB}$ between the samples. This difference attributed to the slightly differing microstructures of each piece of 3D-C, resulting in differences in efficiency. The specific surface area has been changed in the 
theoretical model to tune the power density to the results (1.4 times higher than with 110ppi). Above $20 \mathrm{kHz}$, the diffraction differs slightly in the experimental results but a strong antiresonance is observed in the model. This is explained by the higher thickness of the 130ppi sample and the regularly spaced layers in the foam considered in the model. When each layer radiates acoustically coherent sound waves, each layer would add up coherently with a slight delay

$\sum_{n=0}^{N} \cos \left(k x_{0}+n k \frac{L_{z}}{N}\right)=\cos \left(k x_{0}+k \frac{N}{2} \frac{L_{z}}{N}\right) \frac{\sin \left((N+1) \frac{k L_{Z}}{2 N}\right)}{\sin \left(\frac{k}{2} \frac{L_{Z}}{N}\right)}$.

Anti-resonances then occur at every frequency with $k$ as an integer

$f=\frac{C_{0}}{L_{z} \frac{N+1}{N}} k=\frac{343}{5 \times 10^{-3} \frac{71}{70}}=6.76 \times 10^{4}$,

as observed in Fig. 10 with $k=1$. Other than high frequency diffractions caused by the difference in thicknesses and hence number of radiating layers, both samples perform similarly acoustically. However, the 130ppi sample cools approximately twice as fast as the 110ppi sample. Videos found in Supporting Information (Videos S1 and S2) show that the cooling process of the sample was improved with higher ppi. The increase in ppi resulted in an additional increase in the number of pores in the 3D-C, allowing air to circulate more freely in the material, increasing the rate of cooling by thermal conduction towards the surrounding air. 
(a)

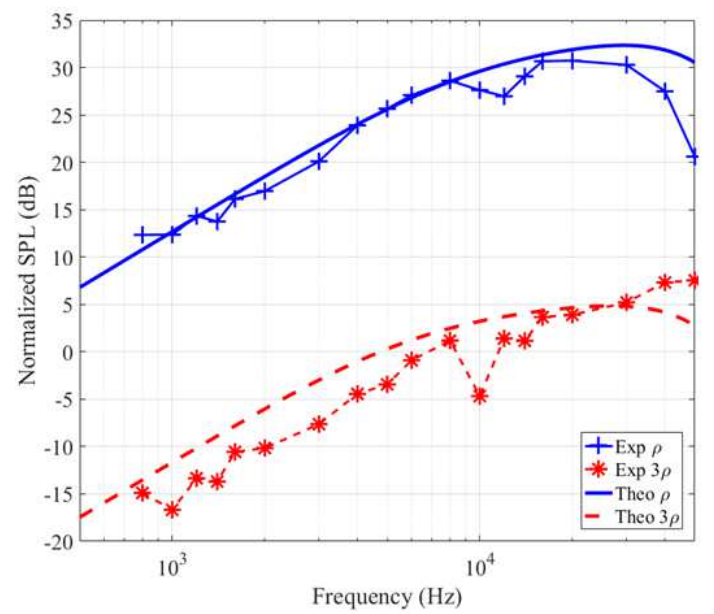

(b)

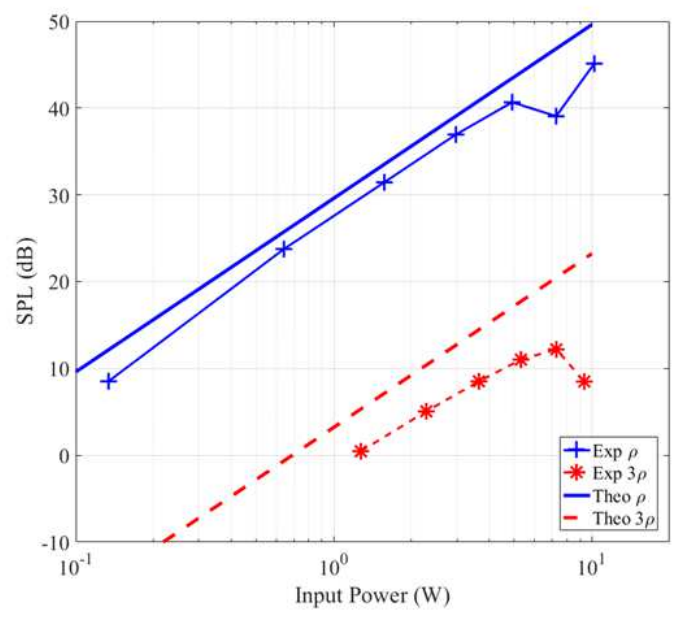

Fig. 11. (a) Acoustical frequency and, (b) power spectrum of 3D-C with different densities.
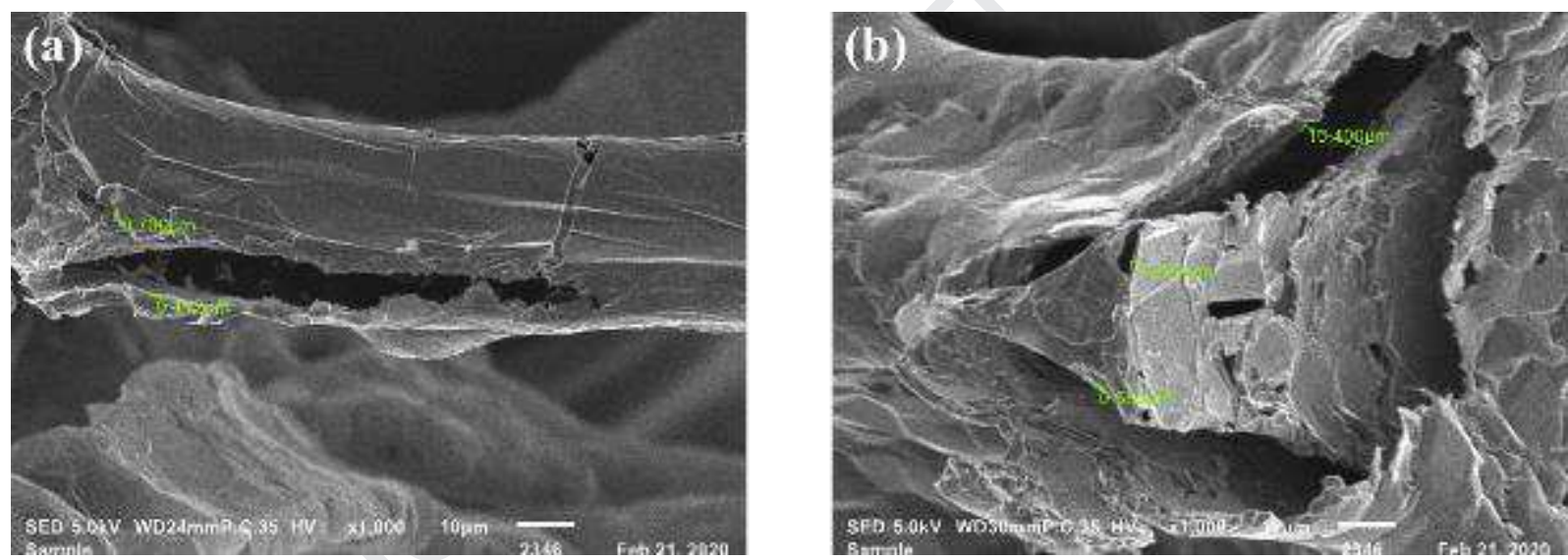

Fig. 12. SEM micrographs of $3 D-C$ taken up close. a) SEM micrograph of $\rho$ sample; b) SEM micrograph of $3 \rho$ sample.

Fig. 11 compares two samples with different densities. Unlike the previous samples, the $3 \rho$ sample had a longer growth time of graphene, leading to thicker branches and a material with three times the density. HCPUA is considered the most important parameter in the global efficiency of thermophones [24,31], which can be written as $\rho C_{p} l$ with $l$ the thickness of the radiating surface. To achieve optimum thermoacoustic radiation, HCPUA has 
to be as low as possible. Increasing the density of the thermophone would then diminish its acoustical performance. Fig. 11a shows both experimental frequency spectra, and a drop of $25 \mathrm{~dB}$ is observed across the whole frequency range for the $3 \rho$ sample. The trends of both curves are similar and the theoretical model fits both curves. This fit was obtained by increasing the theoretical HCPUA value by a factor of $~ 27$. This is justified by the higher measured density, increased thickness of the branches (Fig. 12) and the proportional increase of the specific heat. The power spectrum as seen in Fig. 11b displays the same behaviour as Fig. 11a. The SPL is reduced by 25 to $30 \mathrm{~dB}$ in the $3 \rho$ sample and the increase of HCPUA allows a good fit between theoretical predictions and experimental results. The few $\mathrm{dB}$ differences between theory and experiments of Fig. $11 \mathrm{~b}$ can be explained by the local experimental reduction of the SPL at $10 \mathrm{kHz}$ seen in Fig. 11a.

\subsubsection{Improvement of sound pressure level}

Next, we investigate a method to improve sound generation in 3D-C based on wellknown acoustical results and new experiments on the 3D-C structure. 


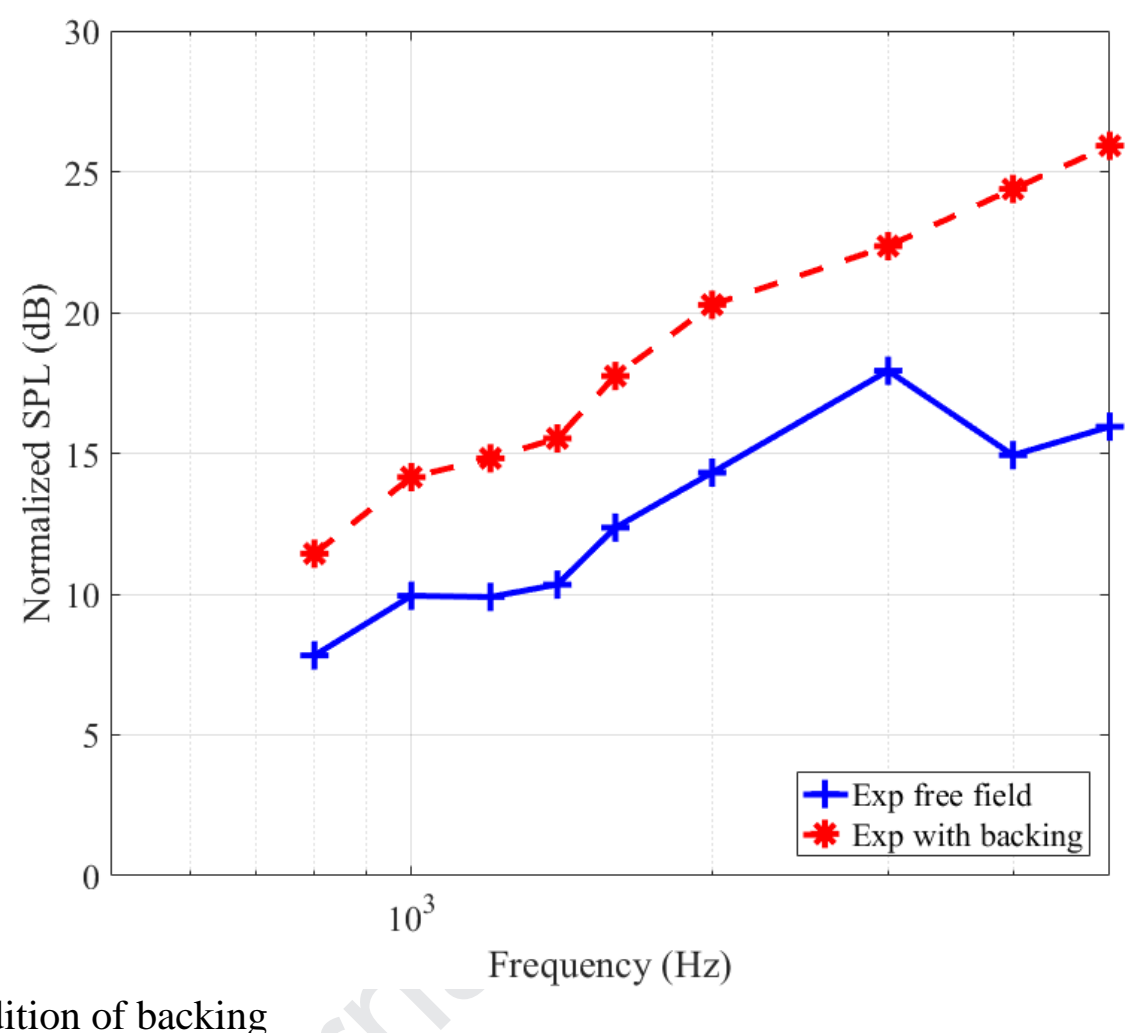

\subsubsection{Addition of backing}

Fig. 13. Acoustical frequency spectra of 3D-C radiating in free field and with an acoustical backing.

In a general acoustic context, when a monopole is placed on a reflecting surface, a coherent reflection from the backing will double the emitted sound pressure, thus causing a $6 \mathrm{~dB}$ increase in the SPL. In theoretical thermoacoustic models, it has been shown that if a thermophone is directly in contact with its backing, some thermal losses will occur. Part of the thermal energy used for the acoustic generation will be lost by thermal conduction in the backing [28, 34-36]. To prevent this, it is crucial to leave a large enough air gap between the sample and the backing. This air gap should be longer than the thermal layer in which the 
thermoacoustic generation occur. It is also important to remember that the thermal layer is inversely proportional to the square root of the frequency (see Eq. (24)). For instance, in air, the thermal layer length is about $\sim 260 \mu \mathrm{m}$ at $100 \mathrm{~Hz}$ and so $\sim 26 \mu \mathrm{m}$ at $10 \mathrm{kHz}$. It is then interesting to note that the size of the pores in $3 \mathrm{D}-\mathrm{C}$ is a few hundred microns, which means that the pore is large enough for the acoustic generation to occur inside of it. In Fig. 13, a sample radiating in free field was recorded, and then an acrylic backing was added $4 \mathrm{~mm}$ behind one side of 3D-C for comparison. As predicted by acoustical theory, the SPL increased by $6 \mathrm{~dB}$ below $5 \mathrm{kHz}$. Above $5 \mathrm{kHz}$ (not shown here), acoustic diffraction caused by the size and thickness of the backing can be observed but is not discussed here as it is a standard acoustic result not related to thermoacoustics. The thermophone performance can hence be improved by $6 \mathrm{~dB}$ by the simple addition of a backing. Nevertheless, this backing has to be placed at a controlled distance: close enough for coherent reflections but at least several hundred microns away from the sample in order to avoid undesired losses by thermal conduction. 
(a)

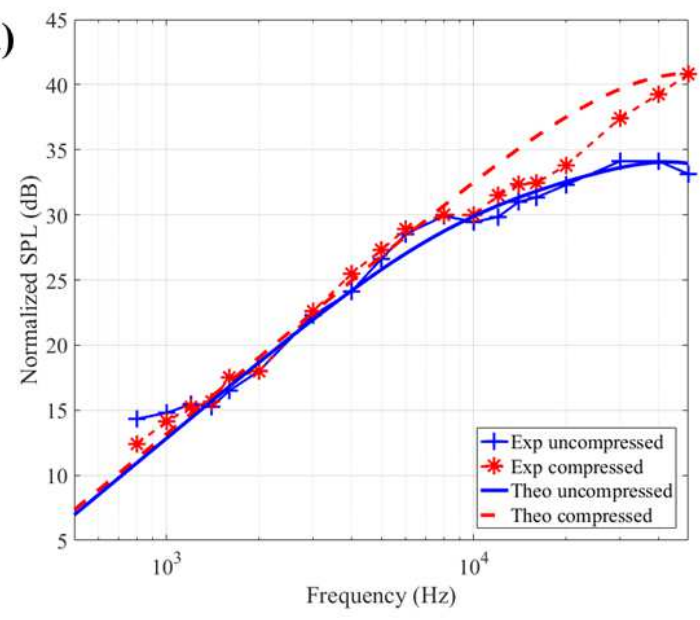

(b)

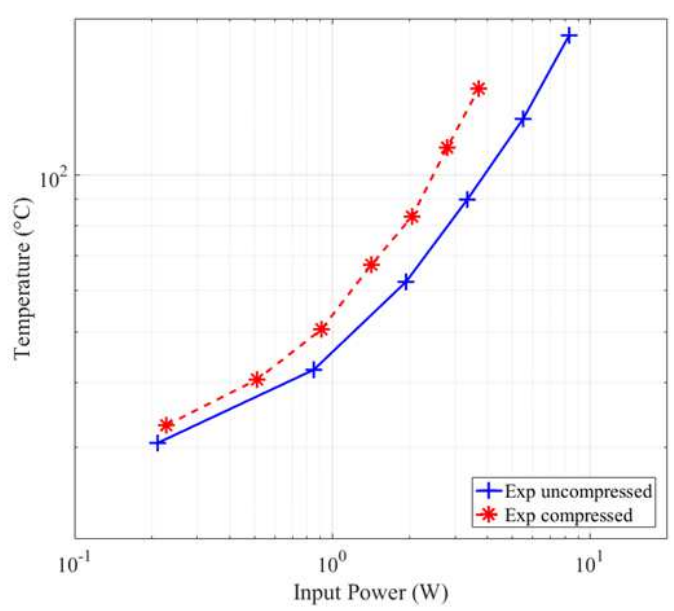

3.2.4.2 Compression of 3D-C material

Fig. 14. (a) Acoustic frequency spectra and (b) temperature dependence of 3D-C before and after mechanical compression, the thickness being $2 \mathrm{~mm}$ and $1 \mathrm{~mm}$ respectively as seen in Table B.2.

The two temperature model proposed in Guiraud et al. 2019 [38] indicated that one method to improve the high frequency radiation of a foam thermophone with a fixed thickness was to increase the number of discretized layers. This would increase the contact surface area between 3D-C and the surrounding air, improving the energy transfer between the two mediums. One way to investigate this property experimentally would be to compare samples with the same thickness and porosity but with a different layer density, a lower ppi. In Fig. 10, the increased thickness compensated the increase in ppi, the number of layers per millimetres did not change (Table B.2). Another way would be to mechanically compress a sample in order to reduce its thickness while preserving its structure, thus artificially increasing the layer 
density more significantly. This would also allow for better comparison if the internal structure of 3D-C is fully preserved after compression. The experimental results of this method are presented in Fig. 14 and are compared with theoretical results in which the number of layers $\mathrm{N}$ is the same in both cases but the thickness reduced, as seen in Table B.2. Fig. 14a shows that at frequencies below $10 \mathrm{kHz}$, the sample acoustically performs similarly before and

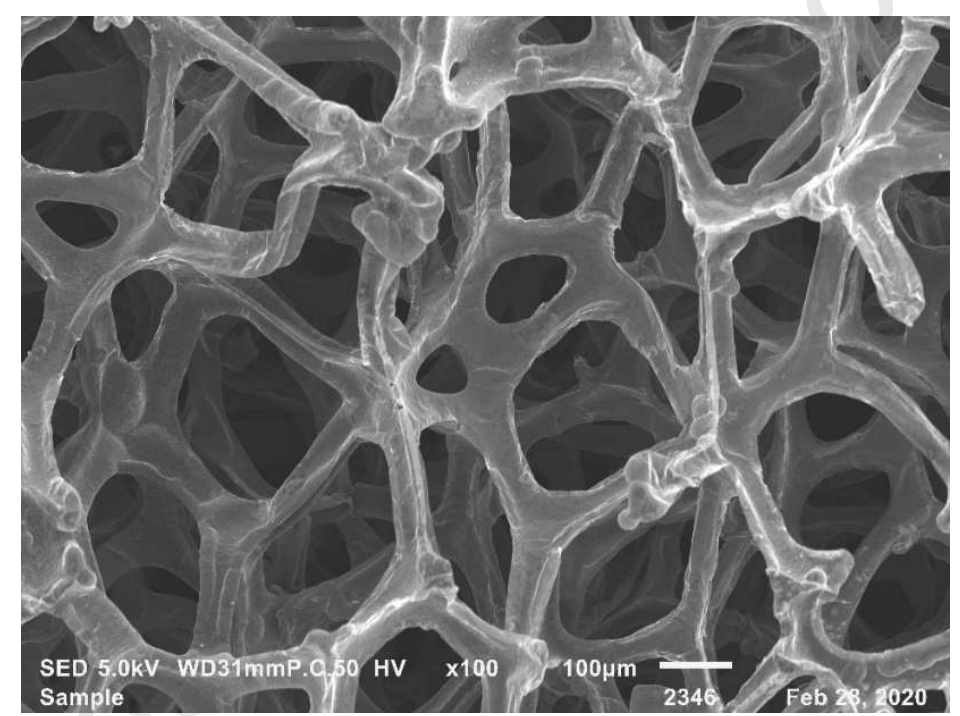

after compression, indicating that the internal structure of the sample was preserved during the compression. This is also proven by the SEM micrograph of the compressed 3D-C in Fig. 15. Fig. 15. SEM micrograph of compressed 3D-C.

Above $10 \mathrm{kHz}$, the maximum SPL of the compressed sample is $6 \mathrm{~dB}$ higher than the maximum SPL of the uncompressed one. The good agreement with the experimental curves confirms that all branches in 3D-C were radiating and that the ppi had been artificially increased by a factor of 2. Considering the theoretical data but ignoring high frequency acoustic diffraction, it is observed that by increasing the number of discretized layers, the SPL 
is linearly proportional to the acoustic frequency up to a higher frequency (slope of $20 \mathrm{~dB} / \mathrm{dec}$ ade up to $40 \mathrm{kHz}$ ). On the other hand, when there is insufficient energy transferred to the air (not enough layers), the SPL would be proportional to the square root of the frequency ( $10 \mathrm{~dB} /$ decade increase for the uncompressed sample from $10 \mathrm{k}$ to $40 \mathrm{kHz}$ ). For practical design of a thermophone device at a designated frequency, it would be interesting to know the minimum number of layers required for maximum efficiency. Due to the complex matrix form

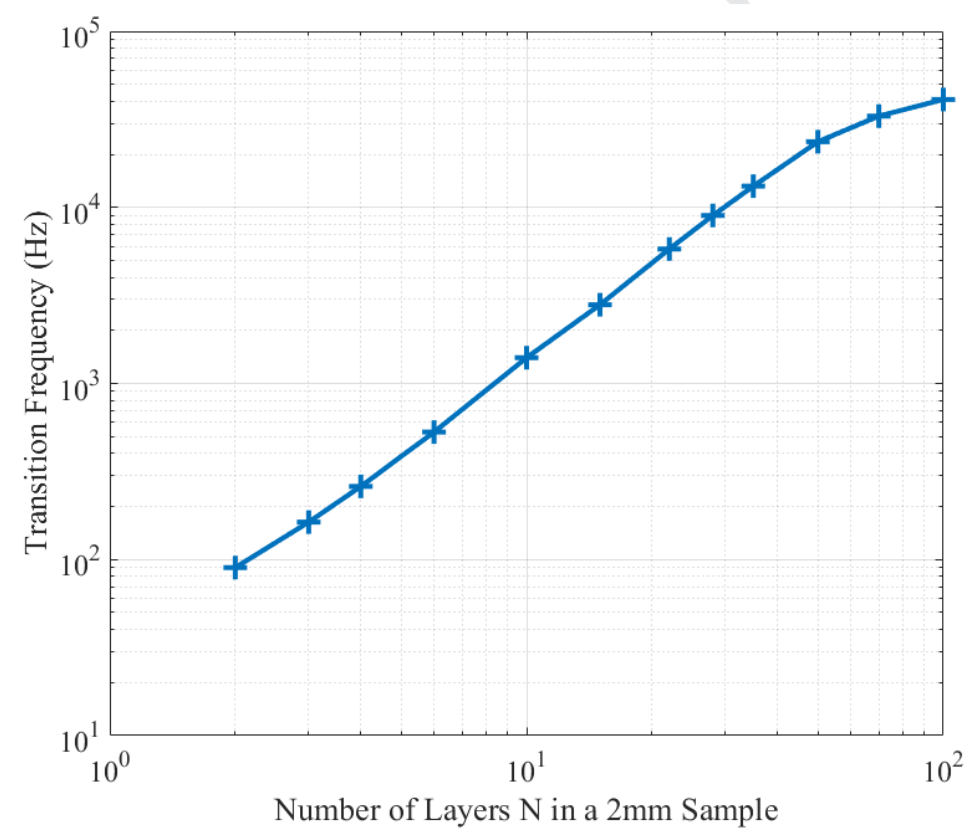

Fig. 16. Transitional frequency depending on the theoretical number of layers in a $2 \mathrm{~mm}$ sample.

of the two temperature model, it is challenging to analytically determine the frequency in which this change of behavior will occur (from 20 to $10 \mathrm{~dB} / \mathrm{dec}$ ) based on the number of layers. A numerical trend can however be observed in Fig. 16. 
Lastly, Fig. 14b showed that at equal input power, the compressed sample had a higher operating temperature. This is attributed by the compression causing the material to be denser,

\begin{tabular}{|c|c|c|c|}
\hline Thermophone & Substrate & SPL (dB) at 1kHz & Reference \\
\hline & & 36 & $\begin{array}{c}\text { Maximum from } \\
\text { Vesterinen [33], see } \\
\text { Eq. (31) }\end{array}$ \\
\hline Freeze Dried 3D-C & Glass & 25 & Fei 2015 [24] \\
\hline Freeze Dried 3D-C & Free-standing & 25 & Lee 2018 [25] \\
\hline Natural Dried 3D-C & Glass & 15 & This work \\
\hline Natural Dried 3D-C & Glass & 12 & Fei 2015 [24] \\
\hline
\end{tabular}

resulting in a air flow to be more difficult and thus reducing its cooling rate.

\subsubsection{Comparison with literature results}

Table I. Comparison of different 3D-C synthesized via TCVD in the literature. The normalized SPL at $1 \mathrm{~m}$ and $1 \mathrm{~W}$ is measured at $1 \mathrm{kHz}$.

Table I compares the thermoacoustic response of 3D-C synthesized from literature methods [26, 27]. Firstly, minimal research has been conducted on 3D-C due to it being discovered in 2011 [47], which is more than 5 years later than CNTs [49] and graphene [50]. Thus, this leaves room for more thorough understanding of 3D-C geometry. Assuming that the heat capacity of the thermophone is negligible, a maximum value for the pressure generated by thermoacoustics was estimated in the literature as $[7,33]$

$p_{r m s}=\frac{f}{2 \sqrt{2} C_{p} T_{0}} \frac{1}{r} P_{i n}$. 
Eq. (31) depends only on parameters of the propagating medium and was added in Table I as a comparison, assuming sound propagation in air. Table I shows that $3 \mathrm{D}-\mathrm{C}$ presented in this paper performed $10 \mathrm{~dB}$ lower than its freeze-dried counterpart from literature, which had been observed in Fei et al. work [24]. However, the naturally dried 3D-C used by Fei et al. performs $3 \mathrm{~dB}$ lower than the presented foam. This is attributed to the improvement made to the TCVD technique, which is reflected in the pristine quality of the $3 \mathrm{D}-\mathrm{C}$ as seen in the Raman spectroscopy with the absence of the defect peak at $1350 \mathrm{~cm}^{-1}$ (Fig. 3c). Overall 3D-C does not reach the maximum theoretical performance for thermophones and different structures like CNTs have been shown to display higher SPL compared to 3D-C. For instance, at 5kHz, 3D-C is a thousand times less efficient than the theoretical maximum from Eq. (31), which is also a thousand times less efficient than a traditional loudspeaker. Additionally, while 3D-C is not as efficient as CNTs, its internal structure allows for a better cooling during operation, rendering it less vulnerable to damage caused by overheating. A more detailed analysis of various thermophones acoustical and thermal performances compared to $3 \mathrm{D}-\mathrm{C}$ is found in the comparison with other carbon nanostructured materials section of Supporting Information.

Eq. (31) is a maximum that was determined based on the assumption of ideal physical properties of a thermophone and not on its geometry. It would be interesting to utilize 3D-C with such flexible synthesis methods to investigate more complex and potentially more efficient thermophones. Unlike CNTs which require the tedious extraction of its strands from its synthesized forest webs [51], 3D-C's simple synthesis method make it a thermophone worth investigating and improving due to its mass manufacturing capabilities [37]. 


\section{Conclusion}

3D-C with varying parameters were synthesized and the acoustics performance studied and compared to theoretical models and experiments from the literature [7, 26, 27, 38]. Results show that there is good fitting between the theoretical models and the experimental data. Namely the effect of electrical input frequency, input power, material size, area of electrical connection, path of electrical connections, material density, material ppi and the presence of a backing on the acoustical performance of the samples were investigated. This study provides insights on how 3D-C should be used for efficient thermoacoustic effect, from the synthesis, to the packaging, depending on the frequency range of interest. Furthermore, it was shown that the real time hot point temperatures were good indicators of when the sample would decompose.

Despite its small size, the high porosity and pristine graphene quality of 3D-C allowed it to exhibit excellent acoustical performance. Its unique geometry also allowed for more rapid cooling as compared to other carbon nanostructured materials in literature. With a sufficiently large air gap between 3D-C and its backing, the addition of an acoustical backing improved the sample's acoustical performance. The electrical contact surface should be as large as possible with electrical paths which allow electrical current to pass through the entire sample for efficient use of the entire material and optimum heat resilience. In this study, a line connection showed to have the best acoustical and thermal performance.

It is within the authors' knowledge that measurements of very low power input (below a few hundred milli-Watts) has not been investigated in depth, as most power spectra in the 
literature are displayed in a linear fashion [7, 24, 52]. More low power measurements have to be performed with different experimental conditions to confirm the linearity between temperature and input power at all power levels. While 3D-C is able to produce complex sound (music) with only AC signals using a pre-processing method [53] (Video S3), more studies have to be done to improve its efficiency before it can compete with regular loudspeakers in the market of audible acoustics generation. Utilization of 3D-C's flexible synthesis could be a way to improve the efficiency by geometrical means. Another idea to further exploit would be to use thermophone mainly for high frequency applications (3D-C was tested up to $150 \mathrm{kHz}$ and other thermophone up to a few $\mathrm{MHz}$ [35]) in order to utilize the linearly increasing efficiency of thermophone with frequency [11, 33].

All in all, thermophones are promising alternative for sound generation. While their current low efficiencies prevent them from replacing commercial loudspeakers, their nonmechanical sound generation mechanism induces a wide frequency band of operating range. In addition, their lightweight, small size and low cost of production make them a novel alternative for sonar applications or ultrasonic transducers. Particularly, 3D-C has an advantage over CNTs for large scale manufacturing as CNTs require a tedious process of using a nano-manipulator to acquire its strands from CNT forests [51]. The ability of 3D-C to emit sound thermoacoustically is hence worth investigating.

\section{Acknowledgements}


This study is supported in part by the Economic Development Board of Singapore under the Industrial Postgraduate Program (EDB-IPP) with Thales Solutions Asia Pte Ltd and Temasek Laboratories@ Nanyang Technological University seed funding. It is also supported by the French DGA (Direction Générale de l'Armement) under the Conventions Industrielles de Formation par la REcherche Program (CIFRE) with Thales Underwater Systems. This study is developed in collaboration with CINTRA, UMI 3288 CNRS/NTU/THALES (Singapore).

\section{References}

[1] F. Li, M.J. Cabral, B. Xu, Z. Cheng, E.C. Dickey, J.M. LeBeau, J. Wang, J. Luo, S. Taylor, W. Hackenberger, L. Bellaiche, Z. Xu, L.-Q. Chen, T.R. Shrout, S. Zhang, Giant piezoelectricity of Sm-doped $P b\left(\mathrm{Mg}_{1 / 3} \mathrm{Nb}_{2 / 3}\right) \mathrm{O}_{3^{-}}$ $\mathrm{PbTiO}_{3}$ single crystals, Science 364(6437) (2019) 264-268.

[2] J. Hlinka, Doubling up piezoelectric performance, Science 364(6437) (2019) 228-229.

[3] P. De Lange, On thermophones, Proceedings of the Royal Society A 91(628) (1915) 239-241.

[4] H.D. Arnold, I.B. Crandall, The Thermophone as a Precision Source of Sound, Physical Review 10(1) (1917) 22-38.

[5] H. Shinoda, T. Nakajima, K. Ueno, N. Koshida, Thermally induced ultrasonic emission from porous silicon, Nature 400(6747) (1999) 853-855.

[6] K. Suzuki, S. Sakakibara, M. Okada, Y. Neo, H. Mimura, Y. Inoue, T. Murata, Study of Carbon-Nanotube Web Thermoacoustic Loud Speakers, Japanese Journal of Applied Physics 50 (2011) 01BJ10.

[7] A.E. Aliev, N.K. Mayo, M. Jung de Andrade, R.O. Robles, S. Fang, R.H. Baughman, M. Zhang, Y. Chen, J.A. Lee, S.J. Kim, Alternative Nanostructures for Thermophones, ACS Nano 9(5) (2015) 4743-4756. 
[8] A.E. Aliev, C. Guthy, M. Zhang, S. Fang, A.A. Zakhidov, J.E. Fischer, R.H. Baughman, Thermal transport in MWCNT sheets and yarns, Carbon 45(15) (2007) 2880-2888.

[9] A.E. Aliev, M.D. Lima, S. Fang, R.H. Baughman, Underwater Sound Generation Using Carbon Nanotube Projectors, Nano Letters 10(7) (2010) 2374-2380.

[10] A.E. Aliev, N.K. Mayo, R.H. Baughman, D. Avirovik, S. Priya, M.R. Zarnetske, J.B. Blottman, Thermal management of thermoacoustic sound projectors using a free-standing carbon nanotube aerogel sheet as a heat source, Nanotechnology 25(40) (2014) 405704.

[11] A.E. Aliev, D. Codoluto, R.H. Baughman, R. Ovalle-Robles, K. Inoue, S.A. Romanov, A.G. Nasibulin, P. Kumar, S. Priya, N.K. Mayo, J.B. Blottman, Thermoacoustic sound projector: exceeding the fundamental efficiency of carbon nanotubes, Nanotechnology 29(32) (2018) 325704.

[12] T. Bouman, A. Barnard, M. Asgarisabet, Experimental quantification of the true efficiency of carbon nanotube thin-film thermophones, The Journal of the Acoustical Society of America 139 (2016) 1353-1363.

[13] A.R. Barnard, D.M. Jenkins, T.A. Brungart, T.M. McDevitt, B.L. Kline, Feasibility of a high-powered carbon nanotube thin-film loudspeaker, The Journal of the Acoustical Society of America 134(3) (2013) EL276EL281.

[14] A.R. Barnard, T.A. Brungart, T.E. McDevitt, A.E. Aliev, D.M. Jenkins, B.L. Kline, R.H. Baughman, Advancements toward a high-power, carbon nanotube, thin-film loudspeaker, Noise Control Engineering Journal 62(5) (2014) 360-367.

[15] M.E. Kozlov, C.S. Haines, J. Oh, M.D. Lima, S. Fang, Sound of carbon nanotube assemblies, Journal of Applied Physics 106(12) (2009) 124311.

[16] L. Xiao, Z. Chen, C. Feng, L. Liu, Z.-Q. Bai, Y. Wang, L. Qian, Y. Zhang, Q. Li, K. Jiang, S. Fan, Flexible, Stretchable, Transparent Carbon Nanotube Thin Film Loudspeakers, Nano Letters 8(12) (2008) 4539-4545.

[17] L. Xiao, P. Liu, L. Liu, Q. Li, Z. Feng, S. Fan, K. Jiang, High frequency response of carbon nanotube thin film speaker in gases, Journal of Applied Physics 110(8) (2011) 084311. 
[18] Y. Wei, X. Lin, K. Jiang, P. Liu, Q. Li, S. Fan, Thermoacoustic Chips with Carbon Nanotube Thin Yarn Arrays, Nano Letters 13(10) (2013) 4795-4801.

[19] L.H. Tong, C.W. Lim, Y.C. Li, Gas-Filled Encapsulated Thermal-Acoustic Transducer, Journal of Vibration and Acoustics 135(5) (2013).

[20] T. Tu, Z.-Y. Ju, Y.-T. Li, G.-Y. Gou, Y. Tian, F. Wu, Z.-Y. Yan, H.-F. Liu, T.-Z. Yang, Y. Yang, H. Tian,

T.-L. Ren, A novel thermal acoustic device based on vertical graphene film, AIP Advances 9(7) (2019) 075302.

[21] K.-R. Lee, S.H. Jang, I. Jung, Acoustic performance of dual-electrode electrostatic sound generators based on CVD graphene on polyimide film, Nanotechnology 29(32) (2018) 325502.

[22] Y. Nakajima, T. Sugimoto, Flexible sound generator based on thermoacoustic effect, SENSORS, 2012 IEEE, Taipei, 2012, pp. 1-4.

[23] C.S. Kim, S.K. Hong, J.-M. Lee, D.-S. Kang, B.J. Cho, J.-W. Choi, Free-Standing Graphene Thermophone on a Polymer-Mesh Substrate, Small 12(2) (2016) 185-189.

[24] H. Tian, T.-L. Ren, D. Xie, Y.-F. Wang, C.-J. Zhou, T.-T. Feng, D. Fu, Y. Yang, P.-G. Peng, L.-G. Wang, L.-T. Liu, Graphene-on-Paper Sound Source Devices, ACS Nano 5(6) (2011) 4878-4885.

[25] J.W. Suk, K. Kirk, Y. Hao, N.A. Hall, R.S. Ruoff, Thermoacoustic Sound Generation from Monolayer Graphene for Transparent and Flexible Sound Sources, Advanced Materials 24(47) (2012) 6342-6347.

[26] W. Fei, J. Zhou, W. Guo, Low-voltage Driven Graphene Foam Thermoacoustic Speaker, Small 11 (2015) $2252-2256$.

[27] K.-R. Lee, S.H. Jang, I. Jung, Analysis of acoustical performance of Bi-layer graphene and graphene-foambased thermoacoustic sound generating devices, Carbon 127 (2018) 13-20.

[28] H. Hu, T. Zhu, J. Xu, Model for thermoacoustic emission from solids, Applied Physics Letters 96(21) (2010) 214101.

[29] H. Hu, Y. Wang, Z. Wang, Wideband flat frequency response of thermo-acoustic emission, Journal of Physics D: Applied Physics 45(34) (2012) 345401. 
[30] H. Hu, D. Wang, Z. Wang, Solution for acoustic field of thermo-acoustic emission from arbitrary source, AIP Advances 4(10) (2014) 107114.

[31] C.W. Lim, L.H. Tong, Y.C. Li, Theory of suspended carbon nanotube thinfilm as a thermal-acoustic source, Journal of Sound and Vibration 332(21) (2013) 5451-5461.

[32] L.H. Tong, C.W. Lim, S.K. Lai, Y.C. Li, Gap separation effect on thermoacoustic wave generation by heated suspended CNT nano-thinfilm, Applied Thermal Engineering 86 (2015) 135-142.

[33] V. Vesterinen, A.O. Niskanen, J. Hassel, P. Helistö, Fundamental Efficiency of Nanothermophones: Modeling and Experiments, Nano Letters 10(12) (2010) 5020-5024.

[34] P. Guiraud, S. Giordano, O. Bou-Matar, P. Pernod, R. Lardat, Multilayer modeling of thermoacoustic sound generation for thermophone analysis and design, Journal of Sound and Vibration 455 (2019) 275-298.

[35] M. Daschewski, R. Boehm, J. Prager, M. Kreutzbruck, A. Harrer, Physics of thermo-acoustic sound generation, Journal of Applied Physics 114(11) (2013) 114903.

[36] P.L. Torraca, L. Larcher, M. Bobinger, P. Pavan, B. Seeber, P. Lugli, Physical modeling and characterization of thermo-acoustic loudspeakers made of silver nano-wire films, Journal of Applied Physics $121(21)(2017) 214502$.

[37] M. Loeblein, Boron nitride carbon foams for various thermal applications, School of Electrical and Electronic Engineering, Nanyang Technological University, Singapore, 2018.

[38] P. Guiraud, S. Giordano, O. Bou-Matar, P. Pernod, R. Lardat, Two temperature model for thermoacoustic sound generation in thick porous thermophones, Journal of Applied Physics 126(16) (2019) 165111.

[39] S. Giordano, F. Manca, Analysis of heterogeneous structures described by the two-temperature model, International Journal of Heat and Mass Transfer 78 (2014) 189-202.

[40] A. d'Hueppe, M. Chandesris, D. Jamet, B. Goyeau, Coupling a two-temperature model and a onetemperature model at a fluid-porous interface, International Journal of Heat and Mass Transfer 55(9) (2012) $2510-2523$.

[41] L.D. Landau, E.M. Lifschitz, Fluid Mechanics, 2 ed., Butterworth-Heinemann1987. 
[42] L.D. Landau, E.M. Lifshitz, Theory of Elasticity, 3 ed., Butterworth-Heinemann1986.

[43] Z.L. Ngoh, F.N. Leong, R.Y. Tay, M.D. Whiteside, S.S. Chng, J.J. Yu, S.H. Tsang, D. Tan, G.I. Ng, E.H.T. Teo, Boron Nitride Coated Three-Dimensional Graphene as an Electrically Insulating Electromagnetic Interference Shield, 2019 IEEE MTT-S International Microwave Workshop Series on Advanced Materials and Processes for RF and THz Applications (IMWS-AMP), 2019, pp. 127-129.

[44] R. Shivakumar, A. Bolker, S.H. Tsang, N. Atar, R. Verker, I. Gouzman, M. Hala, N. Moshe, A. Jones, E. Grossman, T.K. Minton, E.H. Tong Teo, POSS enhanced 3D graphene - Polyimide film for atomic oxygen endurance in Low Earth Orbit space environment, Polymer 191 (2020) 122270.

[45] M. Loeblein, R.Y. Tay, S.H. Tsang, W.B. Ng, E.H.T. Teo, Configurable Three-Dimensional Boron NitrideCarbon Architecture and Its Tunable Electronic Behavior with Stable Thermal Performances, Small 10(15) (2014) 2992-2999.

[46] A.C. Ferrari, D.M. Basko, Raman spectroscopy as a versatile tool for studying the properties of graphene, Nature Nanotechnology 8(4) (2013) 235-246.

[47] Z. Chen, W. Ren, L. Gao, B. Liu, S. Pei, H.-M. Cheng, Three-dimensional flexible and conductive interconnected graphene networks grown by chemical vapour deposition, Nature Materials 10(6) (2011) 424-428. [48] T. Sugimoto, Y. Nakajima, Second harmonic distortion suppression of thermoacoustic transducer using square root circuit, Acoustical Science and Technology 37 (2016) 99-105.

[49] S. Iijima, Helical microtubules of graphitic carbon, Nature 354(6348) (1991) 56-58.

[50] K.S. Novoselov, A.K. Geim, S.V. Morozov, D. Jiang, Y. Zhang, S.V. Dubonos, I.V. Grigorieva, A.A. Firsov, Electric Field Effect in Atomically Thin Carbon Films, Science 306(5696) (2004) 666-669.

[51] C. Zhu, C. Cheng, Y.H. He, L. Wang, T.L. Wong, K.K. Fung, N. Wang, A self-entanglement mechanism for continuous pulling of carbon nanotube yarns, Carbon 49(15) (2011) 4996-5001.

[52] Z. Zhou, W. Xu, Z. Tong, C.W. Lim, X. Xu, Theory and modeling of multi-layer carbon nanotube thin film thermoacoustic transducer, Applied Thermal Engineering 150 (2019) 143-149. 
[53] P. La Torraca, Y. Ricci, M. Bobinger, P. Pavan, L. Larcher, Linearization of thermoacoustic loudspeakers by adaptive predistortion, Sensors and Actuators A: Physical 297 (2019) 111551. 